\title{
Journal of Advances in Information Technology CONTENTS
}

\section{Volume 13, Number 1, February 2022}

Monitoring and Controlling of a Real-Time Ball Beam Fuzzy Predicting Based on PLC Network and Information Technologies

Suppachai Howimanporn, Sasithorn Chookaew, and Chaiyaporn Silawatchananai

Contribution on the Control of Mixed Constrained Discrete Event Systems for a Flexible Workshop Application

Ben Afia Nesrine, Amari Said, and Messaoud Hassani

Patient-Ventilator Asynchrony Detection via Similarity Search Methods

Chenyang Wang, Uwe Aickelin, Ling Luo, Goce Ristanoski, Mark E. Howard, and David Berlowitz

Balanced Weight Joint Geometrical and Statistical Alignment for Unsupervised Domain Adaptation

M. S. Rizal Samsudin, Syed A. R. Abu-Bakar, and Musa M. Mokji

Government Construction Project Budget Prediction Using Machine Learning.

Wuttipong Kusonkhum, Korb Srinavin, Narong Leungbootnak, Preenithi Aksorn, and Tanayut Chaitongrat

Performance of Machine Learning Techniques in Anomaly Detection with Basic Feature Selection Strategy - A

Network Intrusion Detection System .36

Md. Badiuzzaman Pranto, Md. Hasibul Alam Ratul, Md. Mahidur Rahman, Ishrat Jahan Diya, and

Zunayeed-Bin Zahir

Robust Blind Medical Image Watermarking Using Quantization and SIFT with Enhanced Security.....

Tuan Nguyen-Thanh and Thuong Le-Tien

Journeyfy: An Android-Based Mobile Application Utilizing Persuasive Technology for Assisting in Vice Cessation.

Mary Jane C. Samonte, Dan Christopher B. Fandiño, Angelo Luisse S. Gonzales, and Luis Angelo R. Pono

Data Driven Decision Analysis on the Performance of Electronic Companies with TOPSIS Model

Lam Weng Siew, Lam Weng Hoe, Mohd Abidin Bakar, and Lee Pei Fun

Mental Health Analyzer for Depression Detection Based on Textual Analysis.

Pranav Bhat, Alwin Anuse, Rupali Kute, R. S. Bhadade, and Prasad Purnaye

AI-Powered University: Design and Deployment of Robot Assistant for Smart Universities

Thanh-Hiep Nguyen, Duy-Nhat Tran, Doan-Linh Vo, Van-Hung Mai, and Xuan-Quy Dao

Privacy, Security and Policies of the Semantic Web: A Review.

Sana Al Azwari

An Optimized Neural Network Using Genetic Algorithm for Cardiovascular Disease Prediction .95

Jan Carlo T. Arroyo and Allemar Jhone P. Delima

Production Capacity Prediction of Thyristor Based on Fuzzy Neural Network.

Zhi-Wen Xia, Yi-Fei Wang, Ke-Xin Yang, and Li-Jun Jin 